\title{
DEVELOPING SMARTER BATHROOMS FOR ELDERLY AND DISABLED PEOPLE
}

\author{
Mahmoud Ahmed Abdel Salam and Amal Kamal Shams El-din \\ Ain Shams University, Cairo, Egypt. \\ arch.mahmoud.abdelsalam@hotmail.com, amal_ksh@hotmail.com
}

\begin{abstract}
The aging population has gathered a significant and considerable interest by the Governments nowadays beside industry leaders to develop and create new systems for homes to facilitate living in their own homes instead of the health care facilities high cost.

According to the UN data about elderly percentage aged 60 year or over in the more developed regions was $12 \%$ in 1950, rose to $23 \%$ in 2013 and is expected to reach $32 \%$ in

2050. In the less developed regions ${ }^{1}$

Smart bathrooms give the opportunity for disabled to be independent, which will help them gain self-confidence. Smart bathrooms can provide both older adults and people with disabilities with many different types of emergency assistance systems, and techniques that help them to use the bathroom by themselves as possible

The Aim of this research is to study the smart bathroom designs focusing on elderly and disabled people by highlighting new and smart solutions for older adults and people with disabilities to use the bathrooms by themselves as much as possible in their own homes.
\end{abstract}

\section{Keywords : Elderly And Disabled, Intelligent Design, Bathroom Guidelines, Smart Bathroom..}

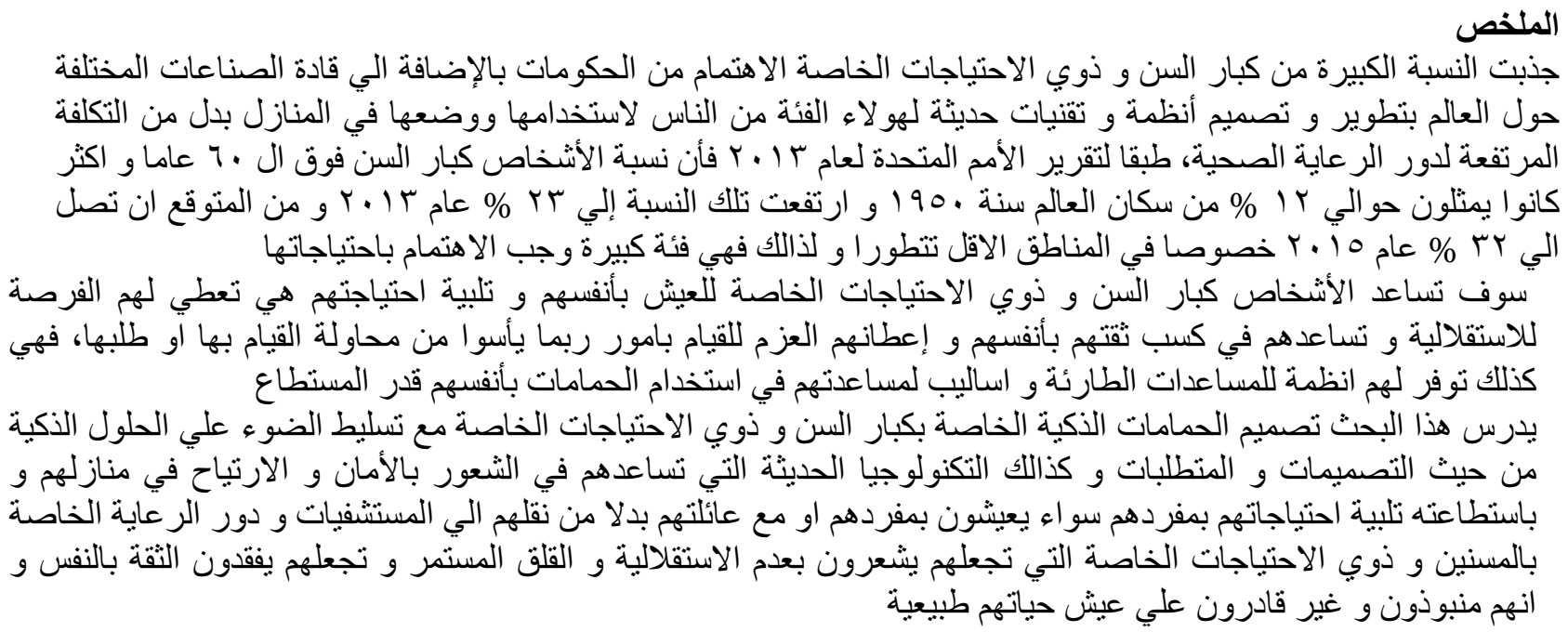




\section{INTRODUCTION}

The number of older persons those aged

60 years or over has increased to a great extent in the recent years in most countries and regions and that growth is expected to accelerate in the coming decades. 2

Between 2015 and 2030, the number of older people aged 60 years or over is expected to grow by $56 \%$ from 901 million to 1.4 billion, and by 2050 is expected to be more than double its number in 2015 reaching 2.1 billion

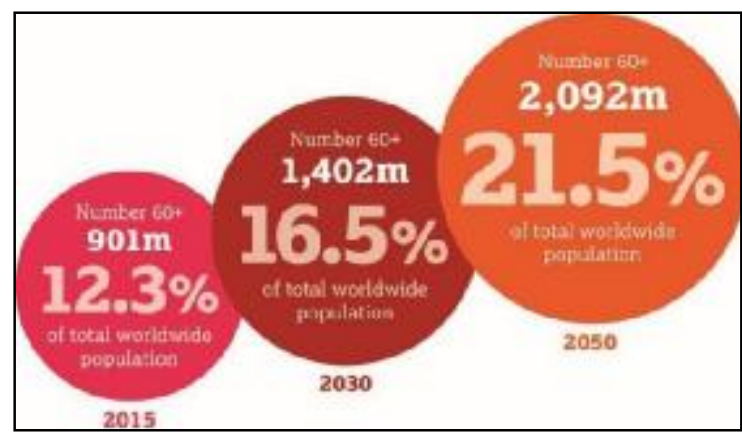

Figure 1: Elderly Percentage expected number Source: World Population Aging Report (2015

The more developed regions were having 38\% of the world's elder people in 2000 and that percentage fell to $33 \%$ in 2015 and is expected to continue to fall to $27 \%$ by 2030 . While the growth rate of the older population in less developed regions increased by $29 \%$ between 2000 and 2015 from 231 million to 299 million and is expected to increase by $26 \%$ in 2030 to reach 375 million, which is less than more developed countries.

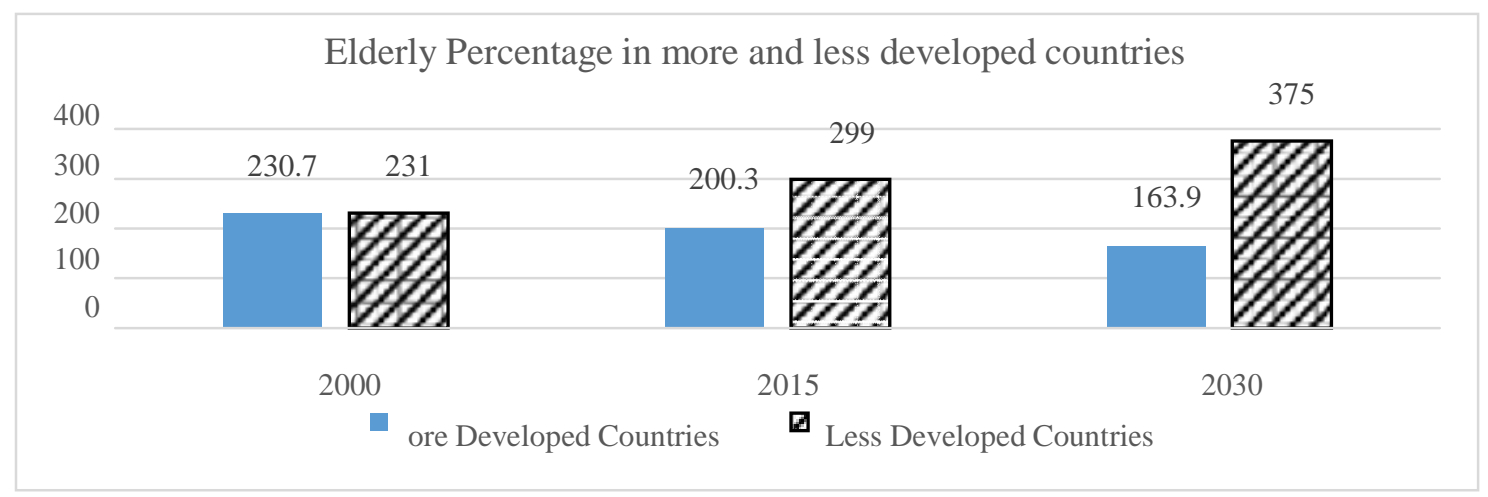

Figure 2: Elderly Percentage in more and less developed countries

Source: World Population Aging Report (2015) 


\section{Problem}

Most bathrooms are designed for healthy and young people who rarely have problem getting their needs done by themselves. But elderly or disabled will face some problem in using the bathrooms by themselves which leads them in the recent future to feel anxious and lose their self-confidence

\section{Objective}

The main objective of the research is to highlight the good design techniques beside smart systems for elderly and disabled in bathrooms and how it can help them to have their needs done by themselves and live their life as before

The other objective is to put the design guidelines for smart homes for elderly and disabled people and how to rehabilitate old homes to support elderly and disabled persons.

\section{Methodology}

For accomplishing the objectives, many steps were used. Based on the objectives that are mentioned above, these steps are:

1. Inductive to highlight the benefits of designing intelligent bathrooms.

2. Comparative to show some examples of design solutions and smart applications for elderly and disabled that can be applied to their bathrooms to facilitate the way they use the bathrooms.

3. Deductive to show the guidelines for designing smarter bathrooms for elderly and disabled persons.

\section{1- Disability}

Disabilities is a general term, covering impairments and weakness, activity limitations, and participation restrictions. An impairment is a problem in body function or structure; an activity limitation is a difficulty encountered by an individual in doing a task or action; while a participation restriction is a problem faced by an individual that is being involved in daily life situations. Disability is thus not just a health problem. It is a complex phenomenon, reflecting the interaction between features of a person's body and features of the society in which he or she lives.

\section{1-1 Disability global problem}

More than one billion people in the world live with some form of disability, of whom nearly 200 million experience considerable difficulties in functioning. This is due to ageing populations and the higher risk of disability in older people as well as the global increase in chronic health conditions such as diabetes, cardiovascular disease, cancer and mental health disorders.

\section{1-2Types of Disabilities}

Disabilities can affect people in different ways, even when one person has the same type of disability as another person. Some disabilities may be hidden, known ${ }_{3}$ as invisible disability. There are many types of disabilities, such as those that affect a person's: ${ }^{3}$

1) Vision Impairment

2) Deaf or hard of hearing

3) Mental health conditions

4) Intellectual disability

5) Acquired brain injury

6) Autism spectrum disorder

7) Physical disability

\section{2- Smart Bathroom Designs \& Applications}

Smart bathrooms for elderly and disabled people require smart solutions oriented designs in addition to the help of some smart devices. Smart design starts from the early stages in designing the building before construction process, where room spaces and design requirements are 
applied according to codes that suits elderly and disabled as mentioned before as well as furniture different designs and its orientation inside the different spaces.

In addition to smart designs, with the help of new technology and devices that are designed to meet different people needs either they were healthy, elderly or disabled to facilitate their way of living besides providing medical care when needed.

Different solutions are found to help the elderly and disabled people in their own homes without the need to go medical facilities with high cost, where it can be added according to their Needs, their financial situation or Medical requirements.

\section{2-1 Bathrooms}

\section{o Design Solutions}

Architectural design solutions should be considered when designing places that can be used by elderly or disabled persons, which can be made in new buildings or to be added in existing buildings.

1- Grab bars are important features to help the elderly get around the bathroom. They can be installed at the shower area and at the toilet for support. (fig.3)

2- To prevent slipping, the bathroom can also be separated into wet and dry area with a shower screen. By keeping the wet area contained and the

washbasin area dry, it is less likely for the elderly to slip and fall if they need to brush their teeth or wash their face. ${ }^{1}$ (fig.4)

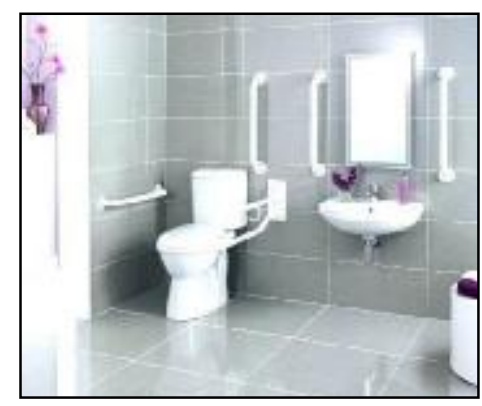

Figure 3: Bathroom grab bars Source: American with Disabilities Act (ADA)

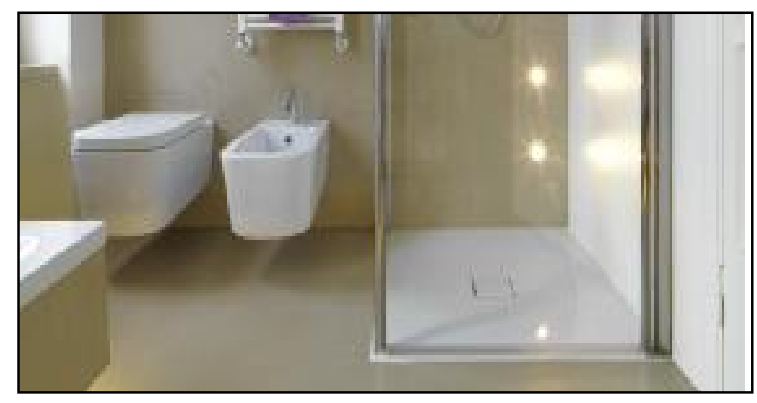

Figure 4: shower area has a glass partition to prevent water from going outside and may lead to falling down. Source: Guidelines for the Planning of Houses for Senior

3- Floor tiles may also be given anti-slip treatment to prevent accidents. Use a rubber or antislip mat if you cannot have the floor treated. (fig.5)

4- Bathroom door should open outwards and not into the bathroom area. If someone falls and is near the door, you can still go in and help. (fig.6)

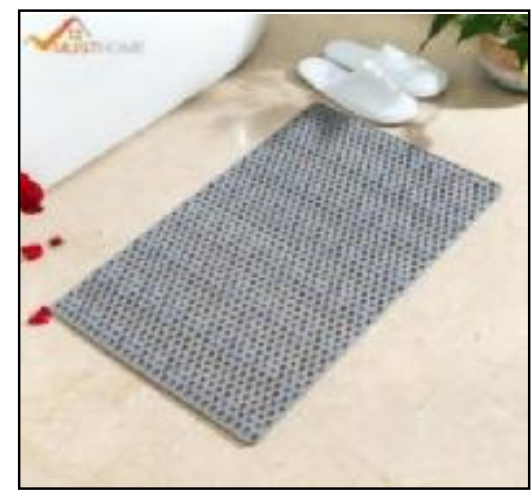

Figure 5: Bathroom anti-slippery floor mat

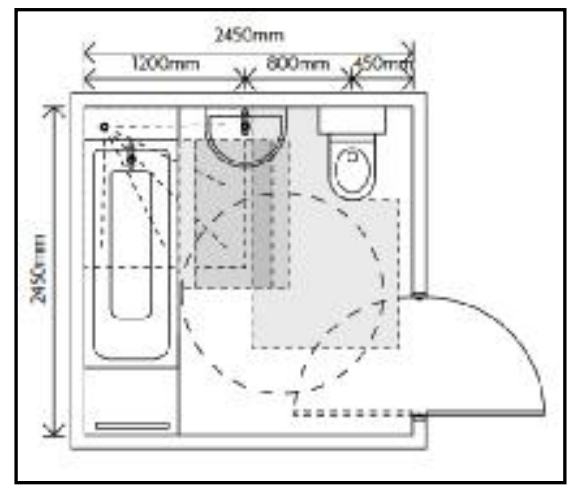

Figure 6: Bathroom door can be opened at both sides. Source: American with Disabilities Act (ADA) 
5- If necessary, put a bath/shower chair so the elderly can bathe in comfort. (fig.7)

6- For the shower, a pressure-balanced lever will prevent any possible scalding. Consider installing a hand-held showerhead in addition to a fixed showerhead as it will be easier for people with limited mobility. (fig.8)

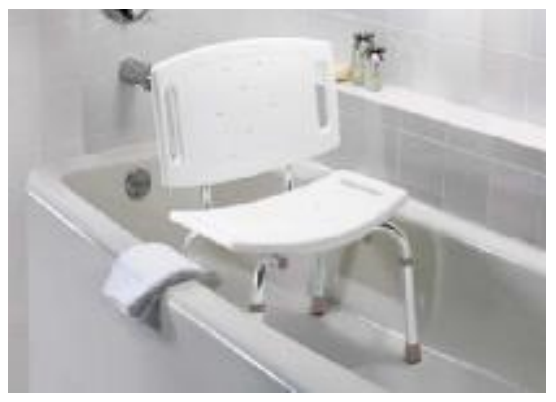

Figure 7: Bathroom chair inside bathtub Source: Guidelines for the Planning of Houses for Senior Citizens, 2007

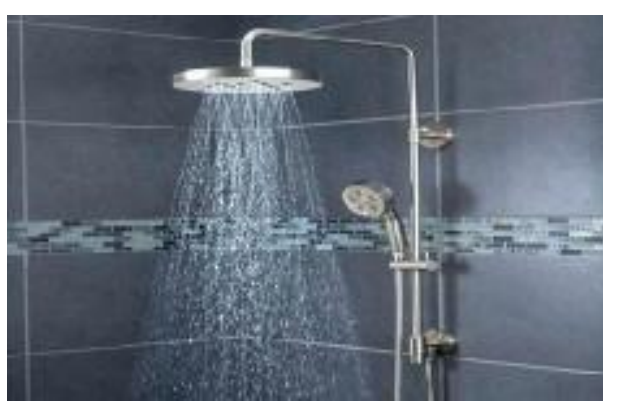

Figure 8: fixed and movable shower head Source: Guidelines for the Planning of Houses for Senior Citizens, 2007

7- It is preferred to add door handle in the bathroom door, which is easily usable even for people with limited manual ability or use sliding doors that are easily to open. ${ }^{1}$ (fig. 9,10 )

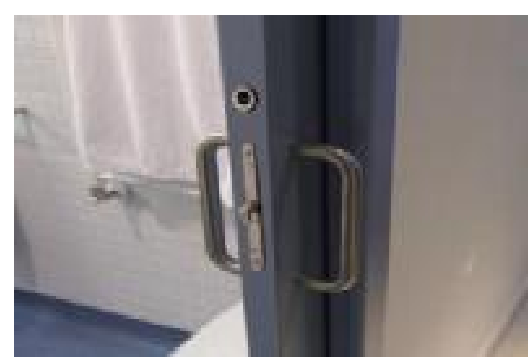

Figure 9: door handles type make it easier for disabled people.

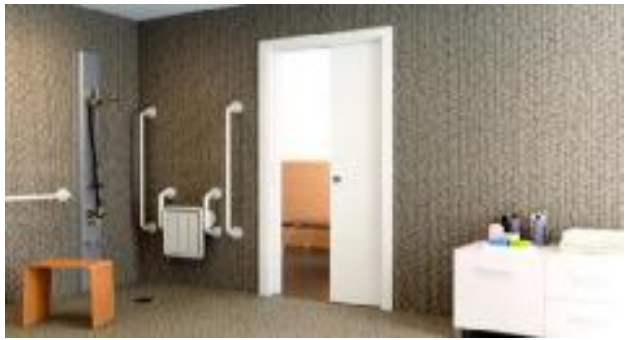

Figure 10: bathroom sliding door

Source: Guidelines for the Planning of Houses for Senior Citizens, 2007

8- Make the bathroom wide enough to allow the complete rotation of a wheelchair (as per ADA Standards). (fig.11)

9- Install a toilet bowl which is easily reached by the lateral transfer.

10- Make sure that the horizontal distance from the toilet bowl to the wall is easy to get up and down (recommended distance is $40 \mathrm{~cm}$ from wall). ${ }^{2}$ (fig.12)

11- Ensure that walls are strong enough to support safety grab bars on both sides of the toilet bowl.

12- Choose a toilet bowl that can be raised or lowered to allow usage for everyone. (fig.13)

13- Install toilet flushing buttons which can be pushed using the whole hand and which requires a little force to push. (fig.14) 


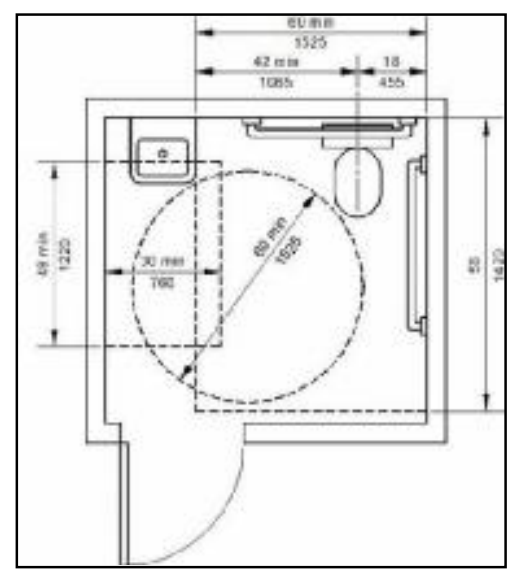

Figure 11: wheelchair rotation diameter Source: ADA standards

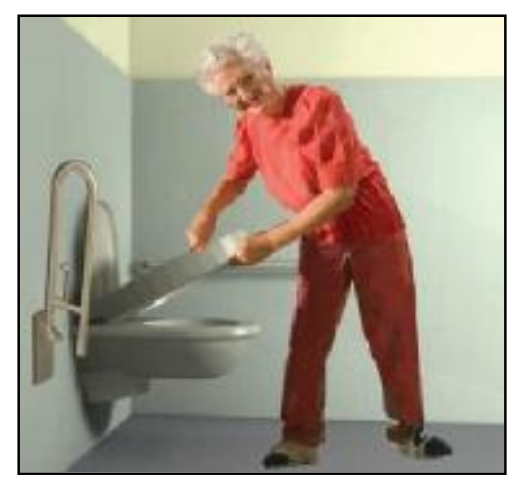

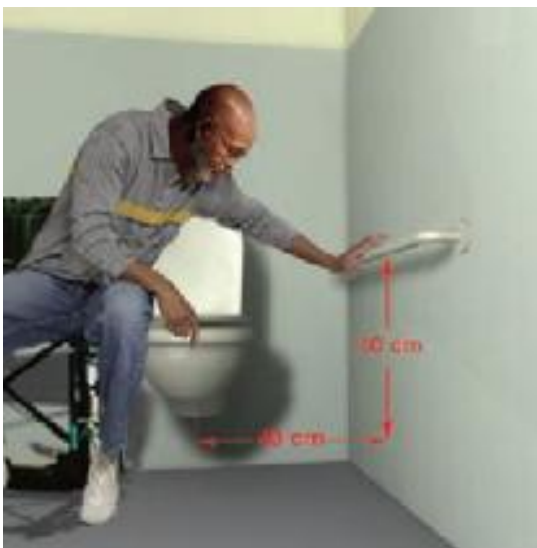

Figure 12: Grab bar distance from wall and toilet Source: Guidelines for the Planning of Houses for Senior Citizens, 2007

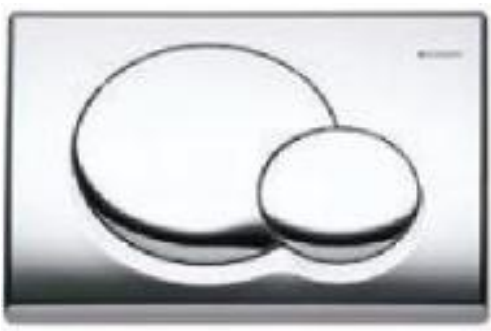

Figure 14: Flushing buttons are easily to use

Figure 13: raised and lowered movable toilet bowl that can. Source:

Guidelines for the Planning of Houses for Senior Citizens, 2007

14- Provide a wash basin which is easily accessible, pedestal basins should be avoided. ${ }^{1}$ 15-Mirrors should be at a height to match a seated person to be able to see him/herself easily.

16- Washbasin tabs should be of a "large cross" type or "handle" to be usable with people with impaired dexterity. ${ }^{2}$

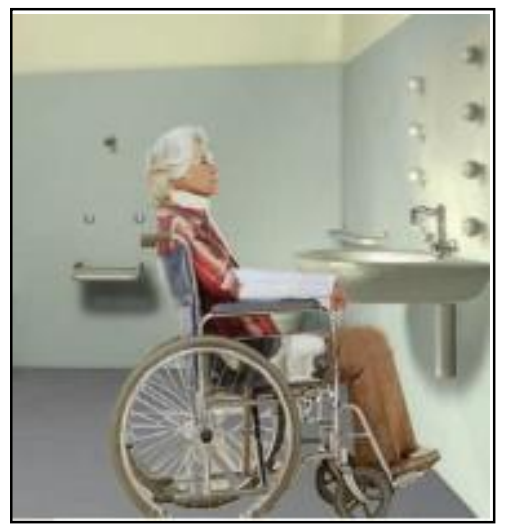

Figure 15: using wall hang washbasin with appropriate height Source: Guidelines for the Planning of Figure 16: large cross handle for the wash basin Houses for Senior Citizens, 2007

JAUES, 14, 52, 2019

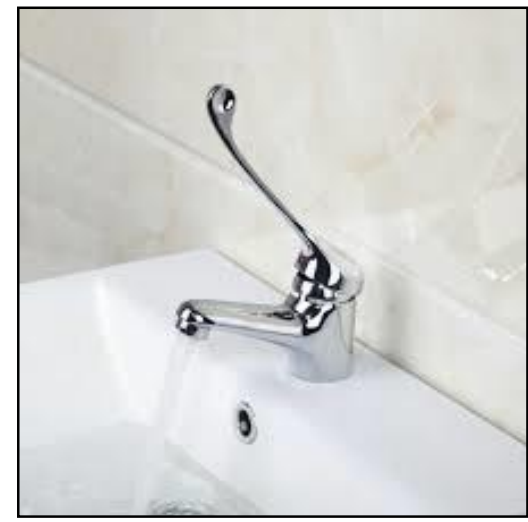

Figure 16: large cross handle for the wash basi 


\section{o Smart Application and Technology}

1- Install emergency aids such as a call bell

2- The call bell should be equipped with a water-proof pushbutton or pull-chord that enables the elderly to activate the bell, it should also be in a color that contrasts with its background. ${ }^{1}$

3- Touchless bathroom water faucet is another good solution for both saving water and can help those disabled who may find it difficult to turn the handles. It works only by putting your hands under the faucet and the photocell sensor gives the signal to the faucet to work and pour water as long as the hands is still under the faucet.

\section{4- Lift and Care Systems}

There are new modern smart systems that made it easier to move around the home for disabled

people using a ceiling rail and motor which has many benefits compared with wheelchair:

a) Motor and rail systems make it possible to access to all areas.

b) Ergonomic lifting with no loss of space.

c) Can be used independently or with the help of someone.

d) Can lift up weight up to $200 \mathrm{Kg} .{ }^{2}$

e) Able to transfer from wheel chair to bed.

f) Automatic and Manual stop at any desired spot

g) Wireless control optional h) Suitable for intensive use

i)Emergency down and stop within reach.

j)Can be used in bathrooms efficiently.

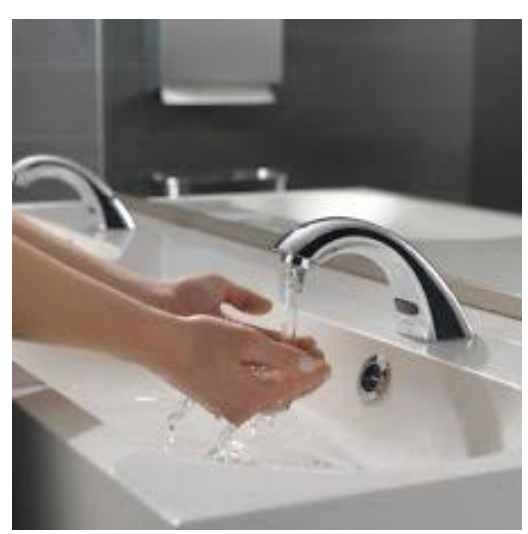

Figure 18: Bathroom touchless faucet

Source: $\underline{w w w . u s . k o h l e r . c o m}$

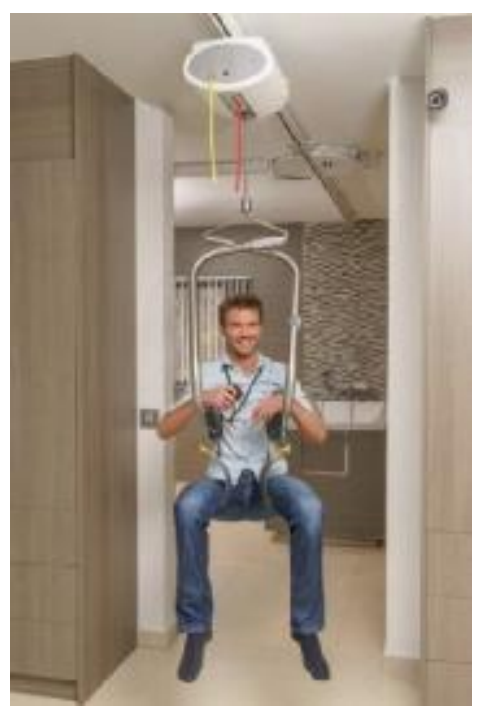

Figure 19: "Handimove" lifting device moving around home spaces Source: www.handimove.com

\footnotetext{
${ }^{1}$ https://www.renonation.sg/how-to-design-an-elderly-friendly-home/
} 
o With the Handi-Move Body Support and ceiling system, some can transfer between bed, bath and toilet with little or no assistance. (fig.20)

o This device can help those who have problem in moving or going to the toilet by themselves either elderly or disabled to be transferred from bed to the bath/shower or toilet using the "Handi-move lift system".

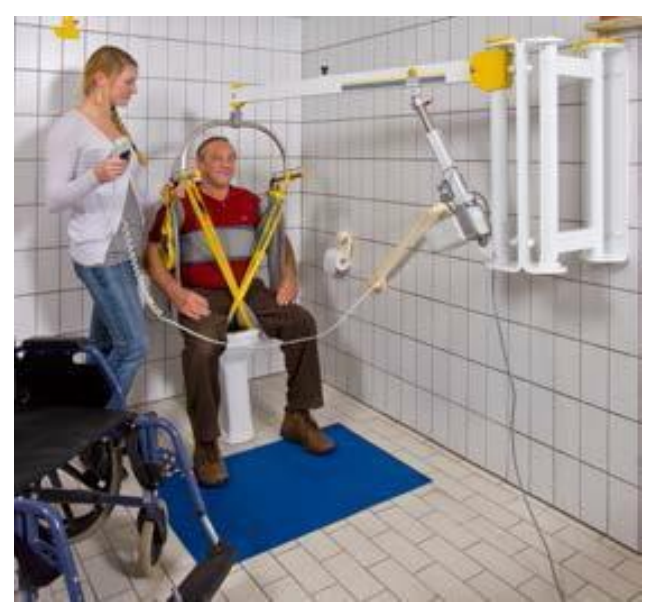

Figure 20: Handi-move lifting system for bathrooms

Source: www.handimove.com

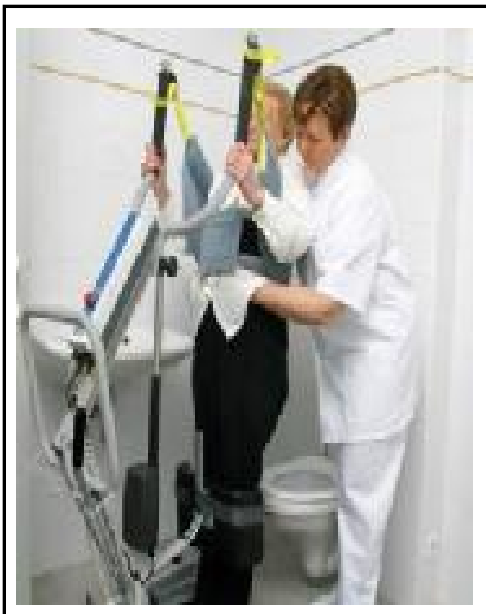

Figure 21 a:

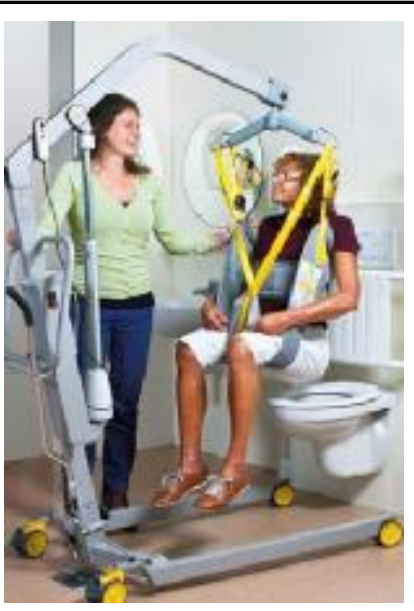

Figure $21 b$ :

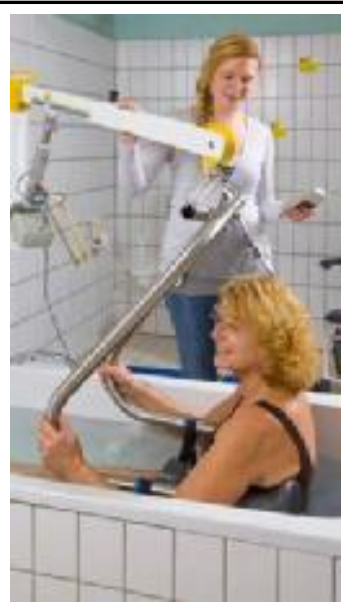

Figure 21 :

Figure 21: Different scenarios for using Handi-move lifting system in the bathrooms Source: $\underline{w w w . h a n d i m o v e . c o m}$

${ }^{1}$ https://www.handimove.com/products/ceiling-motor/\#highlights 
The "Handimove" railing system can be installed on different spaces around home to be easier to move around for doing daily tasks. ${ }^{1}$

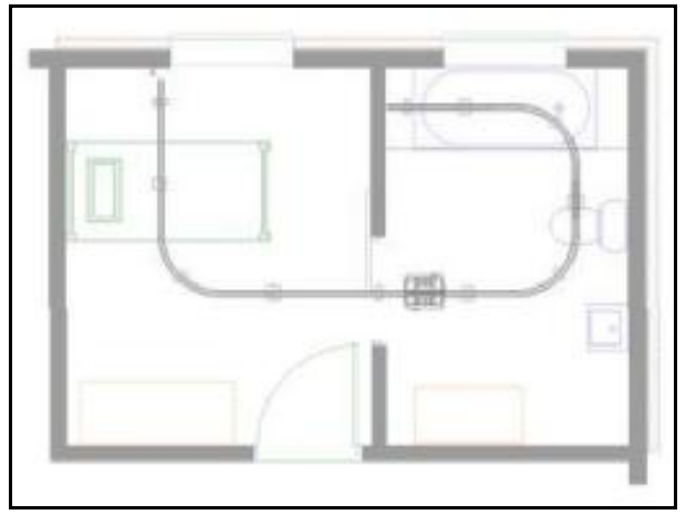

Figure 22: "Handimove" railing plan Example 1 Source: $\underline{w w w . h a n d i m o v e . c o m}$

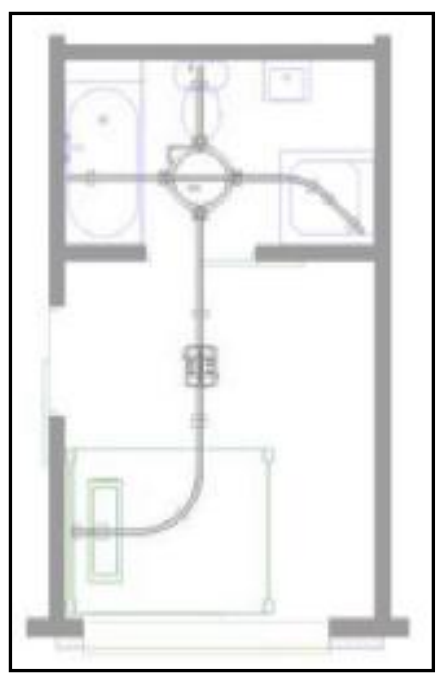

Figure23: "Handimove" railing plan Example 2

Source: www.handimove.com

\section{5- Suction shower handle - Bathroom balance bar:}

It is a safety handrail support for bathtub that is removable and easy to use, it can be used by (Handicapped - Elderly - injury - kids). ${ }^{2}$

\section{Benefits of using Removable grab bars:}

$\square$ Grab bar is easy to install or remove from the tub or shower walls with locking latches to secure bar in place

$\square$ Easy to adjust position on tub or shower: lift tabs allow the grab bar to be repositioned easily. Adjust the height on the shower wall, change to the side of the tub and even take it with you when travelling for a safe bath or shower anywhere.

$\square$ Sturdy and Secure: Strong suction cup design works on any non-porous, flat surface, including tile, glass, acrylic and fiberglass. Supporting up to 300 pounds, the grab bar provides additional stability when entering and exiting the tub or shower for a safe and comfortable experience.

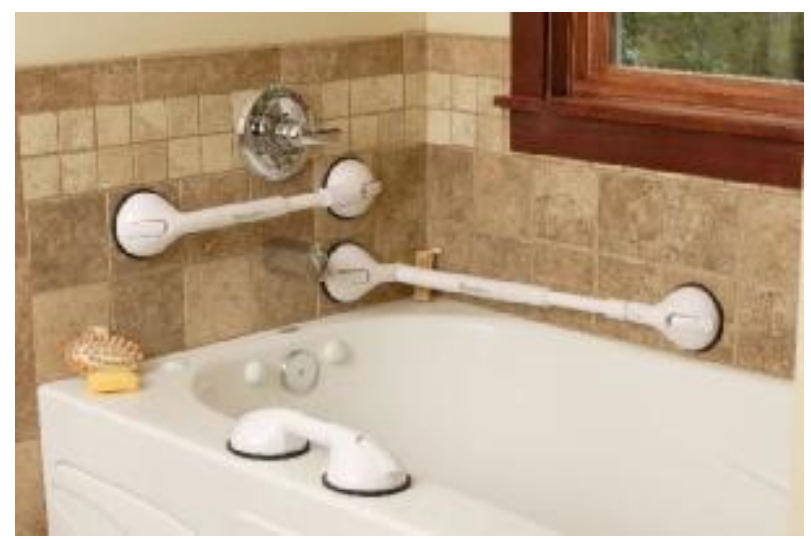

Figure 24: Different scenarios for suction grab bars in the bathrooms with different sizes Source: $w$ ww.vivehealth.com

\footnotetext{
${ }^{1}$ Previous Reference

${ }^{2}$ https://www.vivehealth.com/collections/grab-bars/products/shower-grab-bars 


\section{6- Bathroom Smart Mirror}

A Smart Mirror is like any other Mirror but it is manufactured with a lot of HardwareTechnology like temperature sensors, LED for illumination, LCD display for information, internet connection, touch capabilities, and the brain in these kind of mirrors is the internet connection which controls everything, see figure (24).

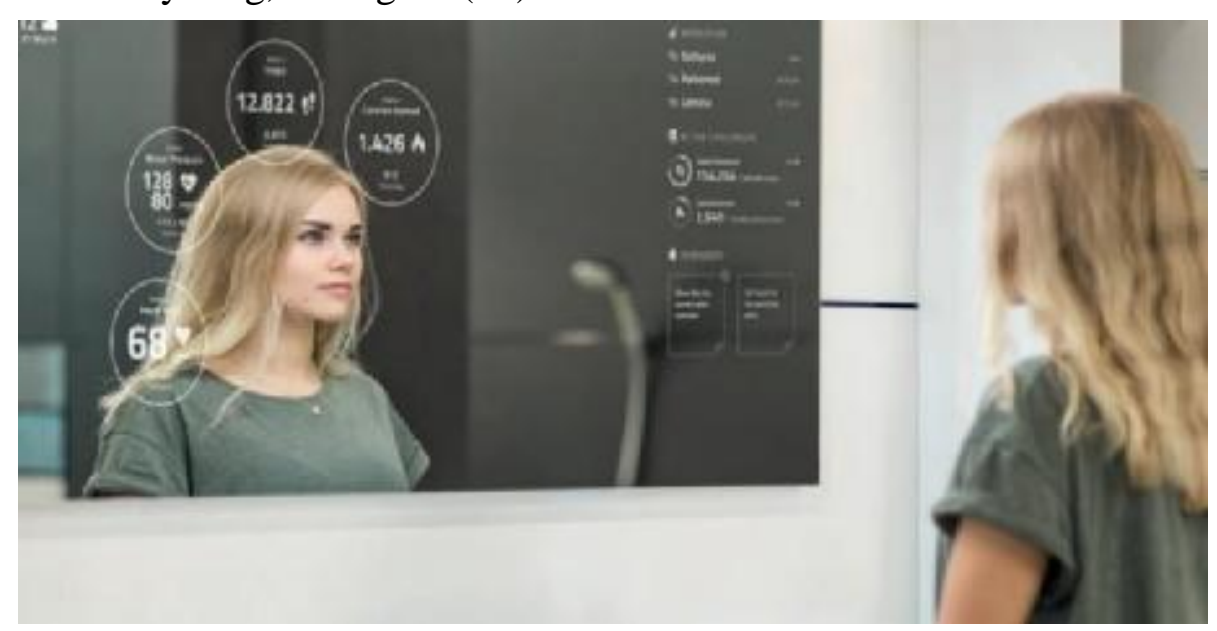

Figure 25: Smart mirrors can detect user's face Source: www.seura.com

Smart mirrors allows you to see all the important information that you need to start your day when you look in the mirror, like the weather information, emails, calendar, estimated traffic, medicine reminders and also your health conditions from your face. ${ }^{1}$

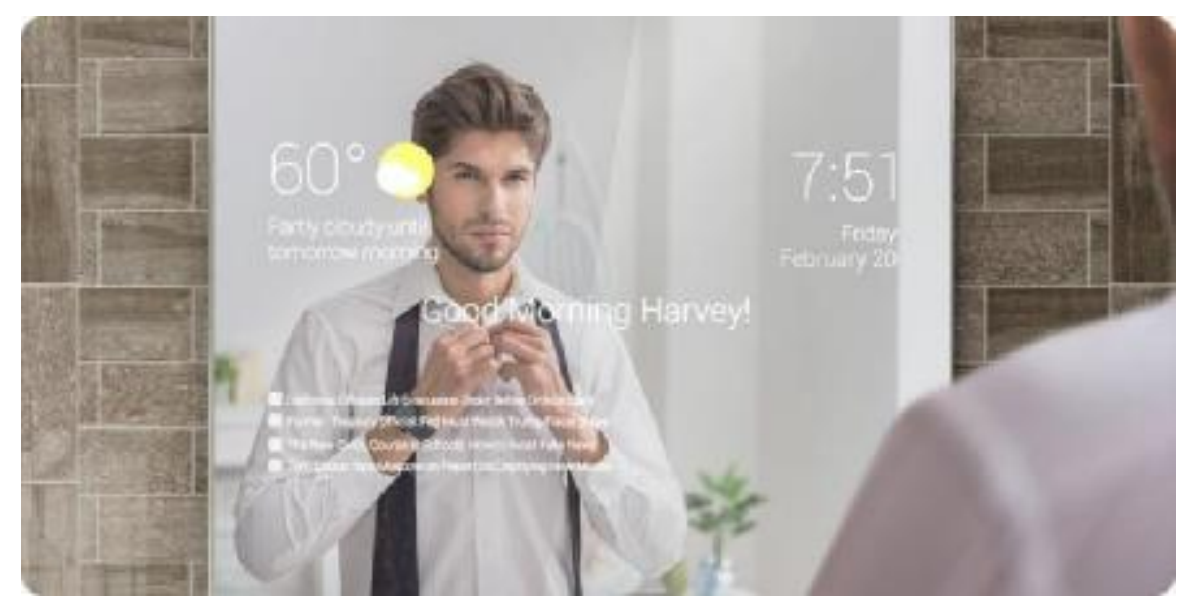

Figure 26: Smart mirrors displaying important meetings and weather data.

Source: www.seura.com

t“What is a smart Mirror?, what can it do for us?" https://medium.com/@ Mauricio.Ingvar/what-is-a-smartmirror-what-can-it-do-for-us-d2b762fc6878 (Mauricio Ingvar, Oct 27, 2017). 


\section{3- ANALYSIS BETWEEN SUITABLE SOLUTIONS AND APPLICATIONS FOR ELDERLY \& DISABLED IN BATHROOMS}

Analysis was conducted to show what the requirements and design guidelines for people with different types of disabilities, elderly or elderly with some form of disability.

Table (1): Analysis between suitable solutions and applications for elderly and disabled in Bathrooms ${ }^{1}$

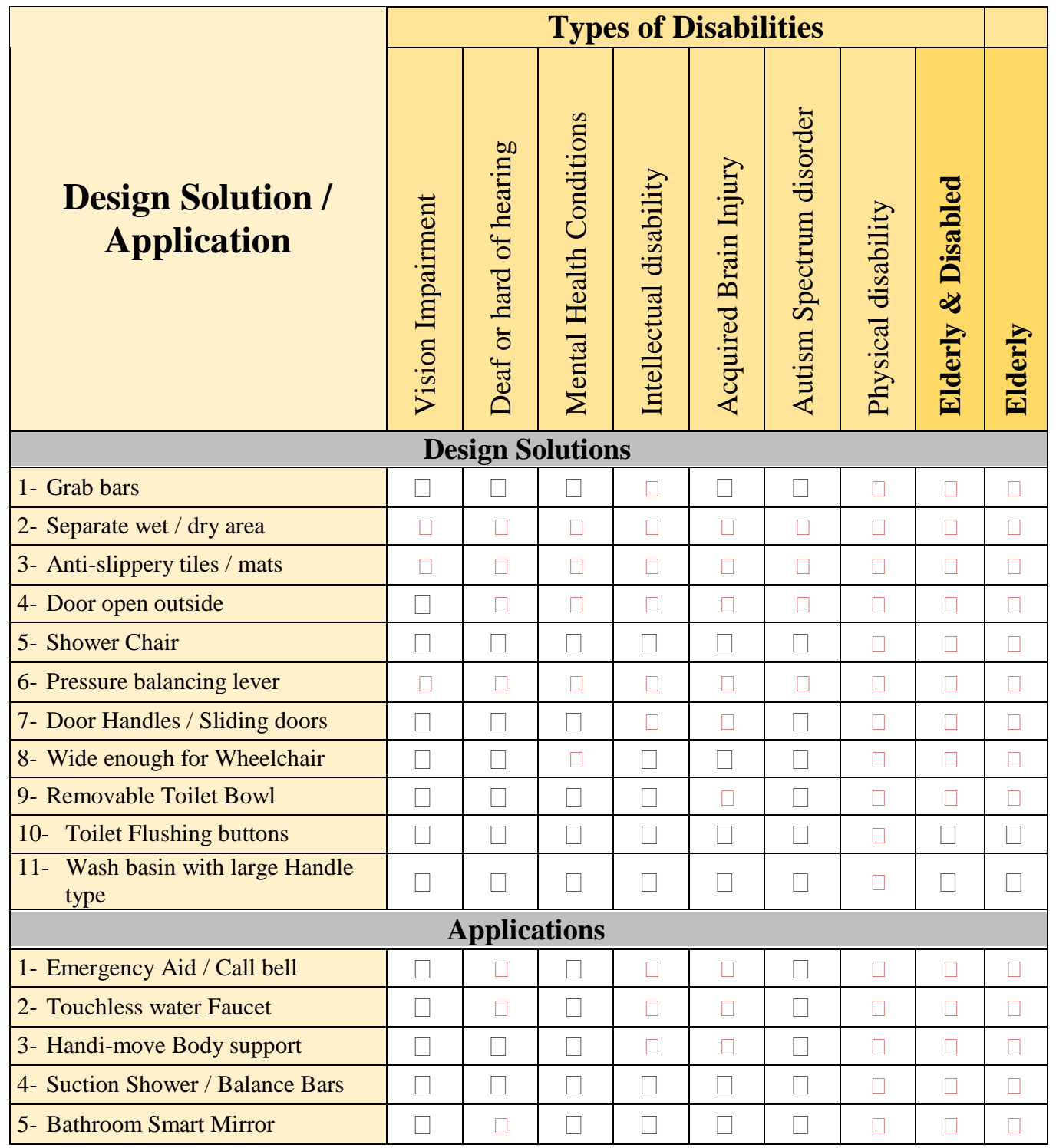

TABLE KEYPLAN
\begin{tabular}{|c|c|}
\hline SYMBOL & MEANING \\
\hline$\square$ & Required \\
\hline$\square$ & Not Required \\
\hline
\end{tabular}

\footnotetext{
${ }^{1}$ The Researcher
} 


\section{4- SUMMARY, CONCLUSION AND RECOMMENDATIO}

$\square$ The number of older persons those aged 60 years or over has increased to a great extent in the recent years in most countries and regions and that growth is expected to accelerate in the coming decades.

$\square$ Two thirds of the world's older persons live in the developing regions and their numbers are growing faster there than in the developed regions.

$\square$ More than a billion people are estimated to live with some form of disability, or about $15 \%$ of the world's population (based on 2010 global population estimates)

$\square$ Many people with disabilities are excluded from decision-making in matters directly affecting their lives.

$\square \mathrm{ADA}$ and Egyptian code requirements for disabled people should be considered in design process and design development procedures to help people who might have any form of disability in the future to deal on their own as much as possible

Living in smart home with hi-tech devices and systems doesn't necessarily mean it will suit all home user's needs, but smart home should be able to be adapted to the needs of its user either with their current health status or with their future needs if someone got any kind of disability or simply grow up and become elderly member that needs special kind of treatment and service.

\section{REFERENCES}

1- United Nations, Department of Economic and Social Affairs, Population Division (2015). World Population Ageing 2015 (ST/ESA/SER.A/390).

2- World Health organization, (2011), "World Report on Disability".

3- Ervet Emillia - Romagna, (2007), Guidelines for the planning of housing for senior citizens, Ohio, United States.

4- World Health organization, (28-10-2016), http://www.who.int/topics/disabilities/en, last accessed

5- The Australian National University, https://services.anu.edu.au/humanresources/respect-inclusion/different-types-of-disabilities

6- Cromly- Home and living, last accessed (3-8-2017), http://stories.cromly.com/stories/Home-andLiving/tips-on- designing-an-elder-friendly-home,

7- Renonation, How to design an Elderly-friendly Home, last accessed (29-4-2014) https://www.renonation.sg/how-to-design-an-elderly-friendly-home/,

8 Handimove, https://www.handimove.com/products/ceiling-motor/\#highlights

9- Vivehealth, https://www.vivehealth.com/collections/grab-bars/products/shower- grab-bars

10- Mauricio Ingvar , , Oct 27, 2017. "What is a smart Mirror?, what can it do for us?" https://medium.com/@Mauricio.Ingvar/what-is-a-smart-mirror-what-can-it-do-for- usd2b762fc6878 last accessed 